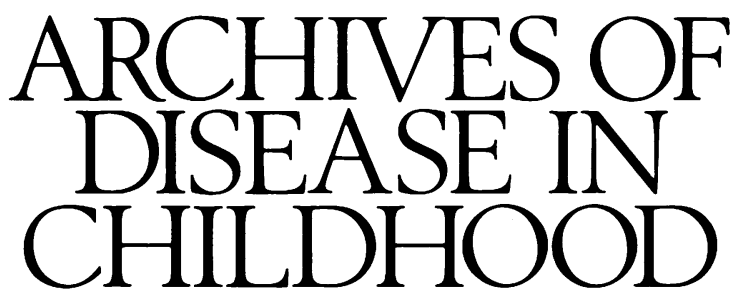

The fournal of the British Paediatric Association

\title{
Annotations
}

\section{Sepsis and intravascular thrombosis}

Platelet and clotting factor abnormalities are often among the first clues to a diagnosis of severe sepsis, and their subsequent progression is widely regarded as an important indicator of poor prognosis. ${ }^{1}$ Although the pathological consequences of the intravascular thrombosis associated with infection have been recognised for over 50 years, ${ }^{23}$ our understanding of the so called disseminated intravascular coagulation syndrome (DIC), how it is triggered, how it is potentiated, and how it may be controlled remains fragmentary. This annotation will focus on the pathogenesis and treatment of the disorder associated with severe bacterial sepsis, examining the new insights that have developed from recent studies of both natural and experimental infection.

For many years DIC has been attributed to the process of thrombin generation leading to fibrin deposition within the microvasculature. Haemostatic failure was then thought to result from secondary platelet activation, consumption of clotting factors, and fibrinolysis. ${ }^{4}$ Early postmortem studies of severe Gram negative sepsis, particularly meningococcal disease, revealed widespread vascular damage, with evidence of vessel wall necrosis, thrombosis and haemorrhage in many organs. ${ }^{256}$ More recently, however, with the recognition that these marked changes are not seen in all septicaemic patients with haemostatic dysfunction, the conventional explanations for the pathogenesis of DIC have had to be re-evaluated.

It appears that in sepsis, the haemostatic disorder results from, and indeed results in, more subtle damage to the vascular endothelium than originally thought. It is now appreciated that the vessel wall is not simply an inert conduit, but performs a number of important homoeostatic functions, including the remarkable ability to maintain blood in an unclotted state. ${ }^{7}$ A detailed description of how the normal endothelium controls the delicate balance between prothrombotic and antithrombotic forces, the factors involved, and their complex interactions is not possible in this annotation. ${ }^{8}$ Briefly, however, coagulation is initiated when either factor XII comes into contact with a surface, activating the kallikrein and the intrinsic coagulation pathways, or when tissue factor comes into contact with the circulation, mobilising both arms of the coagulation system. Recruitment of platelets follows this process. Alternatively, thrombosis may follow platelet activation as a result of surface endothelial changes or the release of leucocyte products such as platelet activating factor (PAF) and cathepsin G. This prothrombotic tendency is counteracted by endothelial anticoagulant molecules such as thrombomodulin and protein $\mathrm{C}$ that inactivate thrombin, and the heparin-like glycosaminoglycans that bind antithrombin III. The surface attachment of

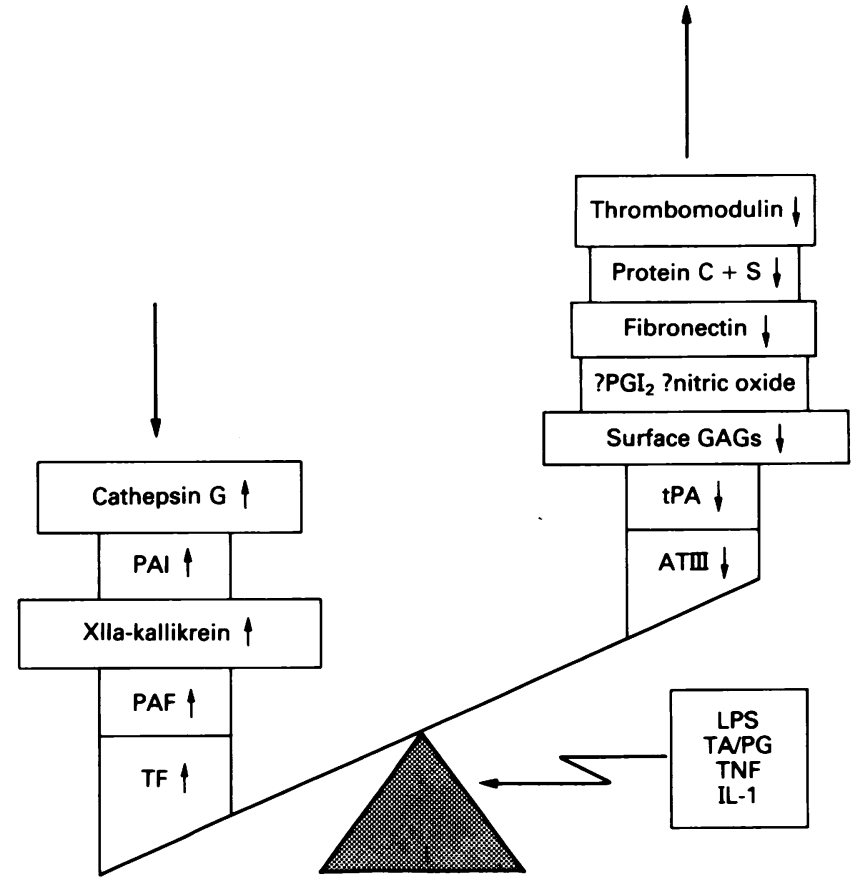

The haemostatic balance in severe sepsis. AT III= antithrombin III, $G A G s=$ glycosaminoglycans, $I L-1=$ interleukin- $1, X I I a-$ kallikrein $=$ the contact system, $L P S=$ =lipopolysaccharide, $P A F=$ platelet activating factor, $P A I=$ plasminogen activator inhibitor, $P G I_{2}=$ prostacyclin, $T A / P G=$ teichoic acid/peptidoglycan, $T F=$ tissue factor, $T N F=$ tumour necrosis factor, $t P A=$ tissue plasminogen.

fibrinolytic agents including tissue plasminogen activator and fibronectin, and the release of endothelial platelet inhibitors such as prostacyclin $\left(\mathrm{PGI}_{2}\right)$ and nitric oxide, further potentiate the antithrombotic process. In sepsis there is a shift in this haemostatic balance (figure), with the elaboration of a number of potent procoagulant, prothrombotic molecules in the face of a deficiency of the natural inhibitors of coagulation. This results in intravascular thrombosis. . $^{-14}$

The precise trigger for the imbalance remains intriguing. Although meningococci, for example, are frequently seen in the characteristic purpuric lesions of the skin, bacteria are very rarely detected in the thrombotic or haemorrhagic areas of other tissues. ${ }^{5}$ Early workers pointed to the similarity between DIC and the generalised Shwartzmann reaction in rabbits, suggesting that in Gram negative sepsis, circulating endotoxin plays a prominent part in initiating both the inflammatory and haemostatic cascades. ${ }^{3}$ More recently it has 
become apparent that in addition to endotoxin and cell wall products from Gram positive organisms (teichoic acid and peptidoglycan) a wide range of inflammatory mediators, including tumour necrosis factor, interleukin-1, and PAF are also capable of altering the haemostatic balance. ${ }^{915} 16$ The relative importance of these agents appears conflicting, and it is likely that their contribution to the disorder is different in individual patients, as well as between different aetiological organisms. In addition, this process is undoubtedly exacerbated by the multiorgan failure that often develops in severe sepsis.

Whatever the trigger, disregulation of haemostasis not only further disrupts normal endothelial function but activates other cellular and humoral systems, such as monocytes and neutrophils, the complement cascade, and the kallikrein-bradykinin system. ${ }^{817} 18$ The relationship between the haemostatic imbalance and the more widespread inflammatory process is therefore central to the pathogenesis of severe infection and has important therapeutic implications.

\section{Management}

How can we switch off this complex cascade of events? The first and most important treatment priority is to eliminate the infecting organism, which involves appropriate antibiotic treatment, and the removal of focal sources of infection. In overwhelming sepsis, however, DIC often persists despite these measures. Currently, infusions of clotting factors in the form of fresh frozen plasma or cryoprecipitate are the mainstay of treatment ${ }^{81019}$ but unfortunately, they are frequently unsatisfactory. Although replacement with purified inhibitors such as antithrombin III, fibronectin, or variants of $\alpha_{1}$-antitrypsin may be more valuable, ${ }^{8120}$ a greater emphasis should be placed on targeting specific treatments to the parts of the imbalance thought to be most affected. A wide range of adjunctive agents have therefore been investigated for their ability to modulate both the inflammatory and haemostatic cascades (see table). This approach appears promising in animal studies but there have been few formal clinical trials, and we can only speculate on what will prove most effective.

Interruption of the inflammatory process should theoretically limit the haemostatic imbalance and prevent further amplification of both systems. Initial attempts using high dose steroids in patients with septic shock were largely disappointing, ${ }^{18}$ with some early studies of meningococcal disease suggesting that they may even worsen the haemostatic picture. ${ }^{3}$ Recently, a number of novel approaches to the modulation of inflammation have been developed which show promise in experimental models and a few small patient

Treatment of the haemostatic disorder associated with severe sepsis

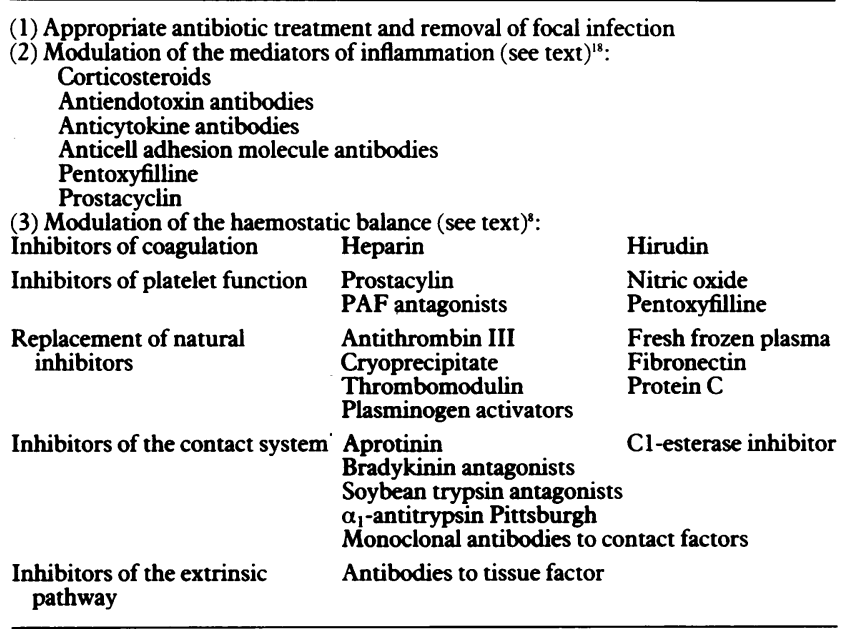

studies (see table). In animals, antiserum to endotoxin prevents the generalised Shwartzmann reaction, ${ }^{21}$ suggesting that antiendotoxin antibodies are a logical therapeutic choice. Antilipid A antibodies have been tested in clinical trials but so far it has been difficult to make firm conclusions. ${ }^{18}$ None the less manipulation of the inflammatory process remains an attractive treatment option and further studies are underway.

Agents designed to specifically treat the haemostatic disorder might be expected to have more success. An immunomodulator, as well as an anticoagulant, heparin has been strongly advocated as rational treatment for DIC. ${ }^{4522}$ In animal models of sepsis, it has been shown to improve survival but has failed to produce obvious benefit in a limited number of open clinical trials, ${ }^{623}$ and has been associated with uncontrolled bleeding. The lack of success with heparin treatment may be explained by the observation that some patients with severe sepsis have circulating glycosaminoglycan-like molecules (R S Heyderman et al, British Paediatric Association 1991 $)^{24}$ that although anticoagulant, fail to prevent thrombosis on the vessel wall. A therapeutic solution to this problem may come from replacement of glycosaminoglycans lost from the endothelial surface during the inflammatory process ${ }^{1425}$ with more antithrombotic but less anticoagulant, heparin or heparin-like molecules.

The prominence of platelet consumption in the evolution of overwhelming sepsis has lead to the clinical use of inhibitors of platelet activation. ${ }^{13}$ Infusions of $\mathrm{PGI}_{2}$ reverse the consequences of endotoxaemia, improve renal perfusion, and ameliorate intravascular thrombosis in animal models. ${ }^{26}$ However, at present the use of $\mathrm{PGI}_{2}$ to treat septicaemic patients with haemostatic dysfunction in largely anecdotal, and further studies are awaited. Nitric oxide (endothelium derived relaxing factor) provides a similar therapeutic approach. Current research has focused on blocking its powerful vasodilatory effects, ${ }^{27}$ but nitric oxide treatment in the form of nitroprusside or nitrate infusions may be helpful in patients with severe vasoconstriction and intravascular thrombosis.

Finally the possibilities for blocking the initiation of the coagulation system are being extensively explored. Antitissue factor monoclonal antibodies and aprotinin (inhibits trypsin, plasmin, and kallikrein) reduce coagulation activation and improve survival in experimental animals. ${ }^{828}$ Aprotinin has been widely employed to decrease the blood transfusion requirements for cardiac surgery, ${ }^{29}$ and more specific inhibitors of the contact system are currently being developed. A combination of agents that block the initiation of coagulation may prove powerful therapeutic tools for the future.

\section{Conclusions}

Subtle changes in the normal haemostatic balance occur early in the course of severe sepsis. There is now increasing evidence that this imbalance results from the disruption of endothelial function, a process that is interlinked with a more widespread inflammatory cascade. It is therefore vital to have an integrated approach to the problem, which includes treatment of the cause, as well as modulation of both the inflammatory and thrombotic processes. This must be coupled with the ability to identify those patients most suitable for treatment, and to intervene at an early stage. The results of trials of specific treatments designed to redress the haemostatic imbalance of severe sepsis are eagerly awaited.

ROBERT S HEYDERMAN

Paediatric Infectious Diseases Unit

Department of Paediatrics,

Queen Elizabeth the Queen Mother Wing,

St Mary's Hospital Medical School,

South Wharf Road, London W2 INY 
1 Gedde-Dahl TW, Bjark P, Høiby EA, Høst JH, Brunn JN. Severity of meningococcal disease: assessment by factors and scores and implications for patient management. Rev Infect Dis 1990; 12:973-91.

2 Ferguson JH, Chapman OD. Fulminating meningococcic infections and the so-called Waterhouse-Friderichsen syndrome. Am f Pathol 1947; 24: 76395 .

3 Margaretten W, McAdams AJ. An appraisal of fulminant meningococcemia with reference to the Shwartzmann phenomenon. Am F Med 1958;25:868-76.

4 Colman RW, Robboy SJ, Minna JD. Disseminated intravascular coagulation (DIC): an approach. Am $\mathcal{F}$ Med 1972; 52: 679-89.

5 Evans RW, Glick B, Kimball F, Lobell $M$. Fatal intravascular consumption coagulopathy in meningococcal sepsis. Am $\mathcal{F}$ Med 1969; 46: $910-8$.

6 McGehee WG, Rapaport SI, Hjort PF. Intravascular coagulation in fulminant meningococcemia. Ann Intern Med 1967; 67: 250-60.

7 Gimbrone MA. Vascular endothelium: nature's blood container. In: Gimbrone MA, ed. Endothelium in hemostasis and thrombosis. Edinburgh: Churchill Livingstone, 1986: 1-12.

8 Bone RC. Modulators of coagulation: a critical appraisal of their role in sepsis. Arch Intern Med 1992; 152: 1381-9.

9 Renesto P, Kadiri C, Chignard M. Combined activation of platelets by cathepsin $\mathrm{G}$ and platelet activating factor, two neutrophil-derived agonists. Cathepsin G and platelet activating

10 Brandtzaeg P, Joø G, Brusletto B, Kierulf P. Plasminogen activator inhibitor 1 and 2, alpha-2-antiplasmin, plasminogen, and endotoxin levels in systemic meningococcal disease. Thromb Res 1990; 57:271-8.

11 Lüscher EF. Activated leukocytes and the hemostatic system. Rev Infect Dis 1987; 9 (suppl 5): S546-52.

12 Fourrier F, Lestavel P, Chopin C, et al. Meningococcemia and purpura fulminans in adults: acute deficiencies of proteins $C$ and $S$ and early treatment with antithrombin III concentrates. Intensive Care Medicine 1990; 16: 121-4.

13 Heyderman RS, Klein NJ, Shennan GI, Levin M. Deficiency of prostacyclin production in meningococcal shock. Arch Dis Child 1991; 66: 1296-9.

14 Heyderman RS, Klein NJ, Shennan GI, Levin M. Modulation of the anticoagulant properties of glycosaminoglycans on the surface of the vascular endothelium by endotoxin and neutrophils: evaluation by an amidolytic assay. Thromb Res 1992; 67: 677-85.

15 van der Poll T, Büller HR, ten Cate H, et al. Activation of coagulation after administration of tumor necrosis factor to normal subjects. $N$ Engl $\mathcal{f}$ Med 1990; 322: 1621-7.

\section{Aetiology of Crohn's disease}

Crohn's disease is an idiopathic inflammation of the gastrointestinal tract occurring anywhere from the mouth to anus, although it is predominantly ileocaecal. It is characteristically patchy, transmural, contains non-caseating granulomas, and has a chronic clinical course with recurrent relapses. It is not seen in infants but can develop in children as young as 4 years of age. Thereafter the incidence increases reaching a peak in late adolescence. It is difficult to determine the exact prevalence of Crohn's disease in the UK but it may be around 50 per 100000 of the population and 10 per 100000 children. Thus Crohn's disease is a significant paediatric problem. Moreover as active disease is associated with diarrhoea, malabsorption, and a general malaise, children with Crohn's disease may be undernourished and a significant proportion are growth impaired. To determine the cause of Crohn's disease is the most significant challenge in gastroenterology today and has been the subject of intense interest over the last 20 years.

Basically, there are three main theories for the cause of Crohn's disease. Firstly, there are those who consider that Crohn's disease is due to an infection, so that it is possible to 'catch' Crohn's disease from an environmental agent; it is however worth pointing out that the epidemiology of Crohn's disease shows no evidence of transmissability. Secondly, there are those who consider that Crohn's disease is due to an immunological hypersensitivity response to antigens (as yet unidentified) in the gut wall. These are most likely to be antigens and components of the normal flora. Thirdly, there are those who consider Crohn's disease to be vasculitis of the submucosal vessels and that mucosal ulceration is a secondary event due to ischaemia. Evidence for these three points of view will be considered. There are also a number of other theories, such as food hypersensitivity or that Crohn's is caused by baker's yeast, but as the evidence for these is scant, they will not be considered here.
16 Girardin E, Grau GE, Dayer J-M, et al. Tumour necrosis factor and interleukin-1 in the serum of children with severe infectious purpura. $N$ Engl f Med 1988; 319: 397-400.

17 Wachtfogel YT, Abrams W, Kuccich U, Weinbaum G, Schapira M, Colman RW. Fibronectin degradation products containing the cytoadhesive tetrapeptide stimulate human neutrophil degranulation. $\mathcal{f}$ Clin Invest 1988; 81: $1310-6$.

18 Jafari HS, McCracken GH. Sepsis and septic shock: a review for clinicians. Pediatr Infect Dis $\mathcal{F}$ 1992; 11 : 739-49.

19 Glynn MFX, Silver E, Redmond MD. A randomised trial of cryoprecipitate replacement of fibronectin deficiencies in the critically ill. Am Rev Respir Dis 1984; 129: A102.

20 Lundsgaard-Hansen P, Doran JE, Rubli E, et al. Purified fibronectin administration to patients with severe abdominal infections: a controlled trial. Ann Surg 1985; 202: 745-9.

21 Braude AI, Douglas H, Davis CE. Treatment and prevention of intravascular coagulation with antiserum to endotoxin. F Infect Dis 1973; 128: S157-64.

22 Corrigan JJ, Ray WL, May N. Changes in the blood coagulation system associated with septicemia. NEngl F Med 1968; 279: 851-6.

23 Gérard P, Moriau M, Bachy A, Malvaux P, De Meyer R. Meningococcal purpura: report of 19 patients treated with heparin. $\mathcal{F}$ Pediatr 1973; 82: $780-6$

24 Zhang CW, Xie WM, Li YF, He ML, Wang ND, Yue JS. Estimation of free heparin in blood and observation of the therapeutic effect of protamine in epidemic hemorrhagic fever. Chung Hua Nei Ko Tsa Chih 1981; 20: 711-5.

25 Klein N, Shennan G, Heyderman R, Levin M. Alteration in glycosaminoglycan metabolism and urface charge on human umbilical vein endothelia cells induced by cytokines, endotoxin and neutrophils. F Cell Sci 1992; 102: 821-32.

26 Ditter H, Matthias FR, Voss R, Lohmann E. Beneficial effects of prostacyclin in a rabbit endotoxin shock model. Thromb Res 1988; 51: 403-15.

27 Nava E, Palmer RMJ, Moncada S. Inhibition of nitric oxide synthesis in septic shock: how much is beneficial? Lancet 1991; 338: 1555-7.

28 Taylor FJ, Chang A, Ruf W, et al. Lethal E coli septic shock is prevented by blocking tissue factor with monoclonal antibody. Circ Shock 1991; 33: 12734

29 Angelini GD, Cooper GJ, Lamarra M, Bryan AJ. Unorthodox use of aprotinin to control life-threatening bleeding after cardiopulmonary bypass. Lancet 1990; 335: 799-800.

\section{Crohn's disease is due to a transmissable environmental agent}

Although various viruses have been reported to be important in Crohn's disease, most work has concentrated on the role of mycobacterial infection. The original description of the disease by Crohn himself focused on the differences between Crohn's disease and intestinal tuberculosis. ${ }^{2}$ Histopathologically there are similarities between the two diseases, but the complete absence of acid fast bacilli in Crohn's disease and the fact that the granulomas in Crohn's disease are noncaseating has generally dampened enthusiasm for this notion. Nevertheless interest in mycobacterial origin of Crohn's disease was rekindled in 1984 by the isolation of an organism, later identified as a slow growing Mycobacterium paratuberculosis from two North American children with Crohn's disease. $^{3}$ This bacterium exists as a cell wall deficient variant in vivo which regains its cell wall after in vitro culture. This organism is of special interest as $M$ paratuberculosis is the causative agent of Johne's disease, a chronic enteritis of cattle. Inoculation of massive amounts of this organism to a goat caused terminal ileitis with non-caseating granulomas, and acid fast bacilli were extremely difficult to detect in the tissues. This however is unlike Johne's disease in cattle in which the mucosa is filled with acid fast bacilli.

In more detailed studies, conventional mycobacteria were isolated from one third of the biopsy specimens of Crohn's disease, $25 \%$ of ulcerative colitis biopsies, and $40 \%$ from controls without inflammatory bowel disease. ${ }^{4}$ These were most commonly of the Mycobacterium avium complex. Only one isolate from a patient with ulcerative colitis was similar to the $M$ paratuberculosis isolated from the children previously. Thus with rigorous culture techniques, mycobacteria can be isolated from the intestinal mucosa of healthy patients and those with inflammatory bowel disease. Cell wall deficient organisms were isolated from a number of patients with 